\title{
Investigation of Ligand Interactions with Human RXR $\alpha$ by Hydrogen/Deuterium Exchange and Mass Spectrometry
}

\author{
Xuguang Yan and Max L. Deinzer \\ Department of Chemistry, Oregon State University, Corvallis, Oregon, USA
}

Michael I. Schimerlik and David Broderick

Department of Biochemistry and Biophysics, Oregon State University, Corvallis, Oregon, USA

Mark E. Leid

College of Pharmacy, Oregon State University, Corvallis, Oregon, USA

Marcia I. Dawson

The Burnham Institute for Medical Research, La Jolla, California, USA

\begin{abstract}
Several different agonists of the retinoic $\mathrm{X}$ receptor $\alpha(\mathrm{h} R \mathrm{X} R \alpha)$ were examined for their effects on the amide H/D exchange kinetics of the homodimeric protein using mass spectrometry. Some agonists, LG 100268, SR11246, and DHA, bind such that slower deuterium exchange-in occurs compared with 9-cis-retinoic acid (9-cis-RA), whereas others, fenretinide and methoprenic acid, result in poorer protection during binding and hence faster exchange-in. Protection against H/D exchange by different agonists and the inhibition of $\mathrm{H} / \mathrm{D}$ exchange kinetics relative to 9-cis-RA varies markedly in different regions of the protein. Agonists LG 100268, SR11246, and DHA generally inhibit faster exchange processes in the ligand binding regions of $h R X R \alpha$ than does the native ligand 9-cis-RA. In at least half of these regions, the level of protection by 9-cis-RA lags behind the agonists even after $60 \mathrm{~min}$. Methoprenic acid did not significantly protect hRXR $\alpha$ against amide hydrogen exchange. An efficient method is described for comparing the effects of different agonists on the protein structure of the agonist-RXR $\alpha$ complex. (J Am Soc Mass Spectrom 2006, 17, 1510-1517) (c) 2006 American Society for Mass Spectrometry
\end{abstract}

$\mathrm{T}$ The retinoid $\mathrm{X}$ receptors (RXRs) belong to a family of nuclear receptors that play important roles in development, metabolism, and cellular homeostasis [1,2]. RXRs are activated by several naturally occurring or synthetic retinoids that, by binding to the ligand-binding domain (LBD), induce conformational changes that cause activator association and initiation of the transcription of the genes regulated by a transcription factor [1, 2]. RXR or its dimeric partner functioning as RXRs can exist and function as homodimers or heterodimers with preferred partners from among other members of the nuclear hormone receptor family [3]. Therefore, RXRs and their ligands have critical roles in modulating many diverse signaling pathways.

Among the three RXR isotypes $(\alpha, \beta$, and $\gamma), \operatorname{RXR} \alpha$ is the primary receptor during development, and its bio-

Published online July 26, 2006

Address reprint requests to Dr. M. L. Deinzer, Department of Chemistry, Oregon State University, 153 Gilbert Hall, Corvallis, OR 94331, USA.

E-mail: Max.Deinzer@oregonstate.edu logical function is crucial for normal cellular regulation [4]. Recently, studies have shown that synthetic RXR agonists may have potential as therapeutics for a range of diseases including cancer, diabetes, cardiovascular disease, and obesity [5-7]. In addition, there is evidence suggesting that highly selective binding by synthetic retinoids to RXRs may reduce adverse effects in the treatment of diseases [8]. Thus, $R X R \alpha$ has emerged as an important therapeutic target for synthetic agonists.

Structural studies have revealed a basic mechanistic picture by which ligands regulate RXR activity, i.e., interaction of ligands leads to large conformational changes in the protein that drive dissociation of corepressor molecules and the recruitment of coactivator molecules by the newly created interaction surface resulting from helix 12 movement $[9,10]$. As shown by the X-ray structure, some synthetic ligands, such as LG 100268, can occupy the RXR binding pocket but cause helix 12 to adopt an altered position compared with 9-cis-RA, so that corepressor release fails in the absence of the coactivators. Experiments using two hybrid anal- 


\section{9-cis-retinoic acid}<smiles>CC1=C(/C=C(\C)C=CC(C)CC(=O)O)C(C)(C)CCC1</smiles>

(4E,6Z,8E)-3,7-dimethyl-9-(2,6,6-trimethylcyclohex-1-enyl)nona-4,6,8-trienoic acid

\section{LG 100268}

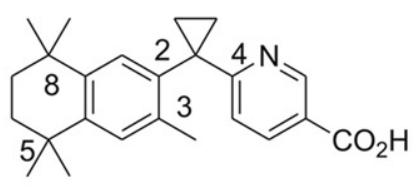

6-[1-(3,5,5,8,8-pentamethyl-5,6,7,8-tetrahydronaphthalen-2-yl)-3-cyclopropyl]nicotinic acid

SR11246

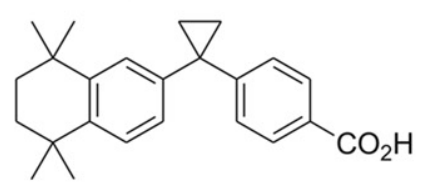

4-[1-(5,5,8,8-tetramethyl-5,6,7,8-tetrahydronaphthalen-2-yl)cyclopropyl]benzoic acid

\section{Fenretinide}

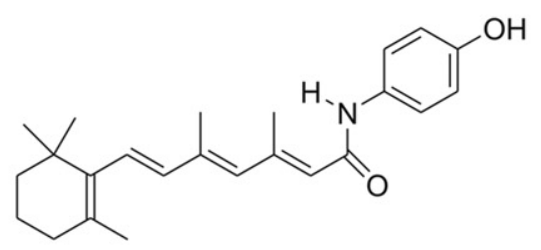

$\mathrm{N}$-(4-hydroxyphenyl)(2E,4E,6E)-3,5-dimethyl-7(2,6,6-trimethylcyclohex-1-enyl)hepta-2,4,6-trienamide

cis-4,7,10,13,16,19-docosahexaenoic acid (DHA)<smiles>CC/C=C\C/C=C\C/C=C\C/C=C\C/C=C\C/C=C\CCC(=O)O</smiles>

(4Z,7Z,10Z,13Z,16Z,19Z)-docosa-4,7,10,13, 16,19-hexaenoic acid

Methoprenic Acid

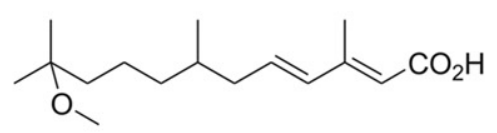

$(2 E, 4 E)$-11-methoxy-3,7,11-trimethyldodeca-2,4-dienoic acid

Figure 1. Chemical structures of the $h R X R \alpha$ LBD agonists. yses [11] suggest that helix 12 is more mobile in the absence of coactivator, and that the coactivator and agonist must bind to stabilize helix 12 in the active conformation. X-ray crystallography and nuclear magnetic resonance (NMR) are well recognized as providing the highest resolution structural information on proteins and protein-ligand complexes, but these methods are limited either because suitable crystals cannot be obtained for X-ray analysis or the amount of protein available is insufficient for characterization by NMR. Hydrogen/deuterium (H/D) exchange coupled with analysis by mass spectrometry is a useful alternative to these methods because the crystalline protein is unnecessary and sensitivity is not nearly the limiting factor that it is with NMR.

A previous study using H/D exchange mass spectrometry on the recombinant human $\mathrm{RXR} \alpha$ ligandbinding domain ( $\mathrm{hRXR} \alpha \mathrm{LBD}$ ) yielded results that were consistent with the information provided by X-ray analysis with regard to the regions of the protein that contact the ligand 9-cis-RA. Furthermore, the H/D exchange mass spectrometric approach provided information that is not obvious from X-ray analysis on the protection of amide hydrogens from exchange in regions that are not directly in contact with bound 9-cis-RA [12]. In the present study, the interactions of the $\mathrm{hRXR} \alpha$ LBD homodimer with several agonists (Figure 1) were explored using H/D exchange mass spectrometry. Agonist-free protein and 9-cis-RA-bound protein were used as controls. The results from these studies may contribute to an understanding of the biological functions and mechanisms of the interaction between the RXR $\alpha$ LBD and these agonists that could be useful in drug discovery and drug development for improved treatments of associated diseases.

\section{Experimental}

\section{Materials and Reagents}

Deuterium oxide $\left(\mathrm{D}_{2} \mathrm{O} ; 99.9 \%\right.$ deuterium), porcine pepsin, and trifluoroacetic acid (TFA) were purchased from Sigma-Aldrich Chemical Co. (St. Louis, $\mathrm{MO})$. 3-[(3-Cholamidopropyl)dimethyl-ammonio]-1propanesulfonate (CHAPS) and tris(2-carboxyethyl) phosphine hydrochloride (TCEP) were obtained from Pierce (Rockford, IN). Poros 20 AL media was purchased from Applied Biosystems (Foster City, CA). The hRXR $\alpha$ agonists, 9-cis retinoic acid (9-cis-RA), methoprenic acid (98\%), cis-4,7,10,13,16,19-docosahexenoic acid (DHA) $(\geq 98 \%)$ were purchased from Sigma-Aldrich Chemical Co., fenretinide ( $\geq 95 \%$ ) was purchased from Tocris (Ellisville, MO). LG 100268 was from Ligand Pharmaceuticals and SR11246 was prepared by one of us (MID). All agonists were prepared as $10 \mathrm{mM}$ stock solutions in ethanol. All other materials were reagent grade and obtained from standard commercial sources.

The $h R X R \alpha$ LBD was expressed and purified as described preciously [12], and confirmed as a ho- 
modimer $(54 \mathrm{kDa}$, in $0.5 \mathrm{M} \mathrm{KCl}, 0.5 \mathrm{mM}$ CHAPS, $1 \mathrm{mM}$ TCEP, and $50 \mathrm{mM}$ potassium phosphate, $\mathrm{pH}$ 7.4) by size-exclusion chromatography and mass spectrometry [12]. The deuterium exchange-in experiments were performed using a homodimer concentration of $45 \mu \mathrm{M}$ (or $\mathrm{hRXR} \alpha$ monomer concentration of $90 \mu \mathrm{M})$ stock solution in aqueous buffer. The buffer $\left(\mathrm{H}_{2} \mathrm{O}\right.$ or $\left.\mathrm{D}_{2} \mathrm{O}\right)$ consisted of $0.5 \mathrm{M} \mathrm{KCl}, 0.5 \mathrm{mM}$ CHAPS, $1 \mathrm{mM}$ TCEP, and $50 \mathrm{mM}$ potassium phosphate buffer ( $\mathrm{pH}$ 7.4).

\section{PDB Structure Analysis}

Protein-ligand contact information was derived using a web site-based program LPC/CSU [13] at http://ligin. weizmann.ac.il/space/servers/ and 3D figures were generated by Pymol. Protein secondary structure was determined by the DSSP program [14].

Sequence alignment analysis was carried out by the web site-based program ClustalW at http://www.ebi. ac.uk/clustalw/. Virtual hydrogen bonding sites were identified using the HBPlus 3.15 program [15].

\section{Hydrogen/Deuterium Exchange and Digestion}

Protein-ligand equilibration was achieved by adding1 $\mu \mathrm{L}$ agonist stock solution $(10 \mathrm{mM}$, or $1 \mu \mathrm{L}$ ethanol as blank) to $35 \mu \mathrm{L}$ homodimer hRXR $\alpha \operatorname{LBD}(45 \mu \mathrm{M})$ and incubating for $30 \mathrm{~min}$ (agonist/monomeric protein molar ratio was over 3:1). The small amount of ethanol $(2.8 \% \mathrm{vol} / \mathrm{vol})$ did not affect protein binding as indicated by $\mathrm{CD}$ titration data. Before deuterium exchangein, the $\mathrm{D}_{2} \mathrm{O}$ buffer, in which the same percentage of agonist and/or ethanol was added, was equilibrated at $30^{\circ} \mathrm{C}$. Deuterium exchange-in was initiated by combining the protein solution $(20 \mu \mathrm{L})$ with $\mathrm{D}_{2} \mathrm{O}$ buffer $(200$ $\mu \mathrm{L})$ at $30^{\circ} \mathrm{C}$. Since the dissociation constants for ligands are in the nanomolar range and the final concentration of binding sites was nearly $10 \mu \mathrm{M}$ after dilution, it was assumed that all ligand binding sites were occupied. After $0.25,1,2,3,5,10,30$, and $60 \mathrm{~min}$, aliquots (20 uL) were taken and added to a prechilled tube containing 20 $\mu \mathrm{L}$ of $0.42 \%$ phosphoric acid to quench the reaction $(\mathrm{pH}$ 2.5). The quenched sample was immediately divided into two tubes and frozen in liquid nitrogen for duplicate LC-ESI-MS analysis later.

The protein was eluted within $6 \mathrm{~min}$ through a C4 column $(0.32 \times 50 \mathrm{~mm}$, Vydac, $10 \mu \mathrm{m}$, in-house packed $)$ with a steep gradient (mobile phase A: $0.04 \%$ TFA in $\mathrm{H}_{2} \mathrm{O}$, mobile phase B: $0.04 \%$ TFA in $\mathrm{CH}_{3} \mathrm{CN}$ ). For intact protein analysis the column was coupled directly to the mass spectrometer. On-line proteolytic digestion was carried out within $3 \mathrm{~min}$ under quench conditions $\left(0^{\circ} \mathrm{C}\right.$ and $\mathrm{pH}$ 2.5), using an in-house prepared immobilized pepsin column as detailed by Wang [16]. The peptic peptides were captured on a $\mathrm{C}_{18}$ trap $(1 \times 8 \mathrm{~mm}$, Michrom Bioresources, Inc., Auburn, CA), then further separated through a $C_{18}$ column $(0.32 \times 100 \mathrm{~mm}$, Luna $\mathrm{C}_{18}(2), 5 \mu$, in-house packed) before delivery into the mass spectrometer within $9 \mathrm{~min}$, using the same elution

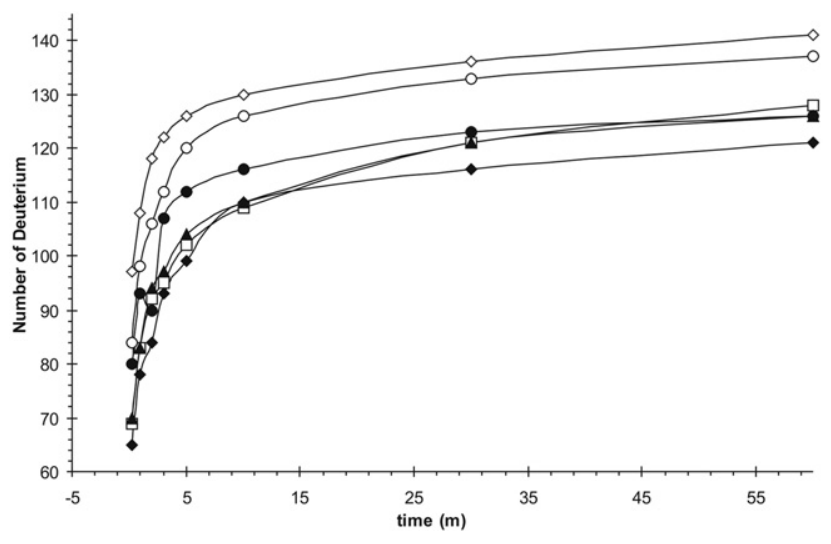

Figure 2. Deuterium levels observed at amide bonds in the subunit of the homodimer $\mathrm{hRXR} \alpha \mathrm{LBD}$ with and without agonist binding as a function of deuterium exchange-in time from 0.25 $\mathrm{min}$ to $60 \mathrm{~min}$ (agonist-free protein, open diamond; 9-cis-RAprotein complex, open square; methoprenic acid-protein complex, open circle; fenretinide-protein complex, filled circle; DHA-protein complex, open triangle; LG 100268-protein complex, filled diamond; protein-SR11246 complex, filled triangle).

solvent as described above. The entire apparatus (injector, enzyme column, $\mathrm{C}_{18}$ trap, $\mathrm{C}_{18}$ column, and part of the solvent transfer tubing for delivery into the mass spectrometer) was immersed in an ice bath.

\section{Mass Analysis and Data Processing}

For MS experiments, spectra on the intact protein were acquired with an LCT mass spectrometer (Waters Corp., Millford, MA) that was equipped with a standard ESI source. All mass spectral measurements were taken with the following settings and conditions: capillary voltage $3 \mathrm{kV}$, sample cone voltage $60 \mathrm{~V}$, extraction cone voltage $5 \mathrm{~V}$, source temperature $80^{\circ} \mathrm{C}$, desolvation temperature $120^{\circ} \mathrm{C}$, desolvation gas $500 \mathrm{~L} / \mathrm{h}$. The intact protein molecular weight (deuterated and nondeuterated sample) was obtained by deconvoluting the combined ESI spectra of the protein with MaxEnt1 of the MassLynx 4.0 program (Waters). The centroided mass of peptic peptides (see below) (deuterated and nondeuterated samples) was calculated from the ESI spectra of the peptide with the Magtran program (Zhongqi Zhang, Amgen, Thousand Oaks, CA). The deuterium levels for protein or peptic peptides were determined from the mass difference between the deuterated and nondeuterated samples. A mass difference of $\leq 0.3 \mathrm{Da}$ was not considered to be a significant difference in $H / D$ exchange in the present study.

Pepsin-generated peptides were identified through the data-dependent MS/MS mode with dynamic exclusion spectra acquired by the LCQ instrument and SEQUEST database search engine against the sequences of the protein and pepsin for assignments. All peptide ion assignments were further verified manually. 

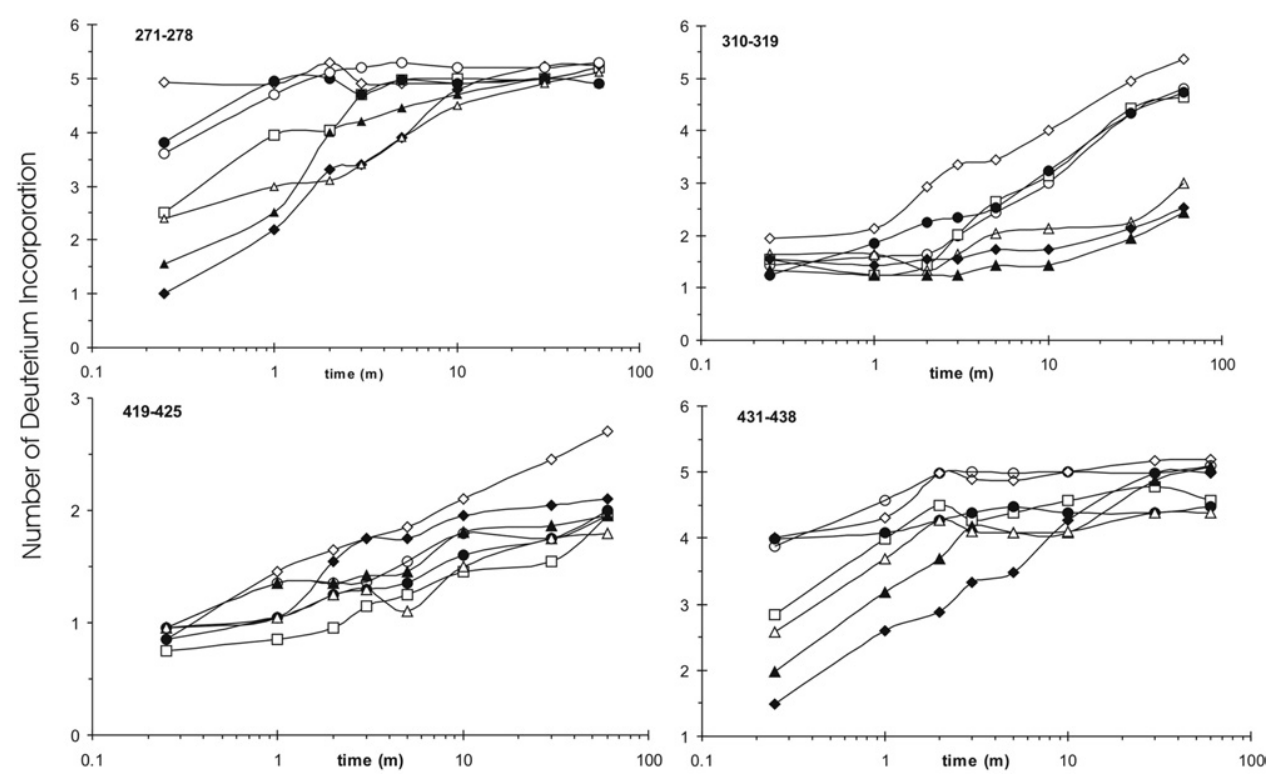

Figure 3. Effects of agonist binding on the deuterium exchange-in rates in fragments corresponding to residues $271-278,310-319,419-425$, and $431-438$ of the protein $h R X R \alpha$ LBD homodimer (agonist-free protein, open diamond; 9-cis-RA-protein complex, open square; protein-SR11246 complex, filled triangle; LG 100268-protein complex, filled diamond; fenretinide-protein complex, filled circle; DHA-protein complex, open triangle; methoprenic acid-protein complex, open circle). No correction was made for proton back-exchange.

\section{Back-Exchange Estimate}

Back-exchange of the hydrogen isotope in both protein and peptic fragments was estimated from the difference of the theoretical mass for the fully deuterated protein or peptides $(0 \%$ loss $)$ and the experimentally measured mass of the fully deuterated sample. The fully deuterated protein was obtained by denaturing the protein in $\mathrm{D}_{2} \mathrm{O}$ buffer containing $7 \mathrm{M}$ urea- $\mathrm{d}_{4}$ and allowing exchange-in for $5 \mathrm{~h}$ at room temperature, and then renaturing the protein in $\mathrm{D}_{2} \mathrm{O}$ buffer by removing urea- $\mathrm{d}_{4}$ using a Biomax membrane ultrafilter (Millipore Corp., Bedford, MA).

It was not considered necessary to adjust for deuterium back-exchange when comparing the effects of various agonists on the same peptide or protein, as the back-exchange should be similar under identical conditions. However, minimizing back-exchange was considered important to avoid losing important deuterium exchange information and to show the greatest possible differences from ligand interactions. In the present study, the back-exchange for the peptides varied between 15 and 38\% depending on their sequence and LC retention time during separation; the average backexchange for the peptides was $29 \%$. The back-exchange for the intact protein was $8.7 \%$.

\section{Results and Discussion}

It was shown previously by H/D-MS and kinetic analysis that the naturally occurring full agonist 9-cis-RA, when bound to the homodimer of $h R X R \alpha$ LBD, exhibited protection against deuterium exchange-in in the ligand binding pocket, mainly within the region of helix 3 , the two $\beta$-sheets, helix 5 , and the carboxyl terminus segment of helix 11. Protection was also found in regions that did not involve direct contact between the protein and the ligand [12]. In the present study, agonists bound to the homodimer of $h R X R \alpha$ LBD triggered similar conformational changes; the interaction specificity was investigated by the same approach. The agonist-free protein and 9-cis-RA-bound protein were used as controls for comparison.

The deuterium level observed in the intact protein as a function of time illustrated a straightforward picture of the influence of these bound agonists on protein backbone amide H/D exchange kinetics (Figure 2). Only a slight protection against deuterium exchange-in was observed for bound methoprenic acid compared with that observed for agonist-free protein, while 9-cisRA-like protection was observed from binding by agonists SR11246 and fenretinide, and greater protection resulted from agonist LG 100268 binding. The mass spectrum from DHA-bound protein that had undergone H/D exchange consisted of diffuse peaks at each charge state, which were not amenable to transformation. From these spectra, no evidence for distinct conformers could be gleaned, but rather a broad nonspecific distribution of deuterium in the DHA-bound protein was observed. Fortunately, the labeled peptides from DHA-RXR $\alpha$ LBD could be analyzed as described below.

The peptic map used in this study was slightly different than the one described previously [12] because digestion was carried out on-line. Nevertheless, se- 
quence coverage was high (95\%). In comparison with the peptic peptides from the agonist-free protein, all investigated agonists except fenretinide and methoprenic acid reduced the deuterium incorporation rate as evident from the analysis of the peptides corresponding to those previously identified from binding of 9-cis-RA [12]. The degree of protection and H/D exchange kinetic profiles, however, varied considerably as illustrated by the results obtained for peptic peptides corresponding to residues $271-278,310-319,419-425$, and 431-438 (Figure 3). The fragment corresponding to residues 271-278 contains three binding residues (Figure 4, bolded AA's) as shown by crystallographic analysis of the 9-cis-RA-protein complex. This fragment exhibited a significant decrease at the early time points (30 $\mathrm{min})$ in the amount of deuterium incorporation when 9-cis-RA bound to the protein in the time course study [12]. Slight protection in deuterium exchange-in was observed for this fragment when agonist fenretinide bound to the protein. The greatest decrease in deuterium incorporation in this fragment occurred on formation of the complex with the agonist LG 100268 or DHA to suggest that more amide hydrogen atoms are protected against exchange-in in the presence of these agonists compared with 9-cis-RA. The observed protection may arise from steric factors or from ligand interaction with specific backbone amide hydrogens; however, it could also arise from an alteration of the protein backbone dynamics in this region. The 9-cis-RA-like reduction in deuterium exchange-in in residues 271-278 in the presence of agonist SR11246 occurred within the
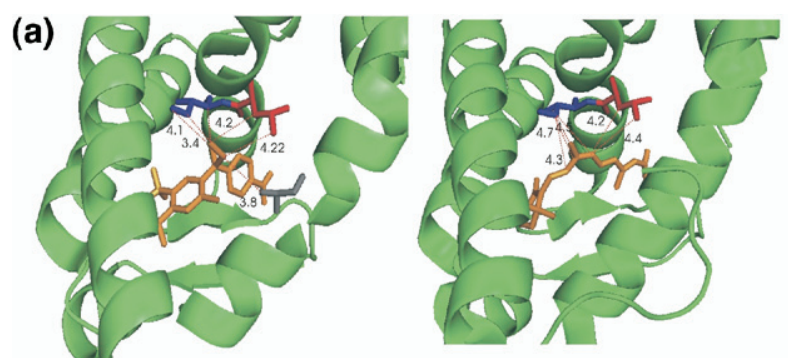

(b)
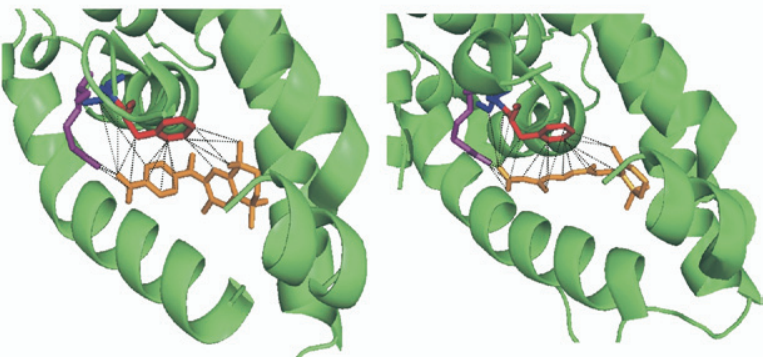

Figure 5. Close-up image of agonist-protein interface in which dashed lines indicate contact atoms. (a) Comparison of hydrophobic interactions of LG 100268 and 9-cis-RA in host protein. The hydrophobic interaction of the cyclopropyl ring in LG 100268 (orange) in the complex hRXR $\beta$-LG 100268 (left) is mainly with Leu380 (red) and Ile381 (blue). The same residues in hRXR $\alpha$ (Leu309 in red, and Ile310 in blue), showing the hydrophobic interaction is mainly provided by the methyl group and C8, C9, and $\mathrm{C} 10$ in 9-cis-RA (orange) in the complex 9-cis-RA-hRXR $\alpha$ (right). (b) Agonist (orange) contacts to Ser383 (red), Phe384 (blue), and Arg387 (purple) in LG 100268-hRXR $\beta$ complex (left), and to Ser312 (red), Phe313 (blue), and Arg316 (purple) in 9-cis$\mathrm{RA}-\mathrm{hRXR} \alpha$ (right) binding pockets. These residues are located in the fragment that corresponds to residues 310-319 in hRXR $\alpha$ LBD, discussed in the text.

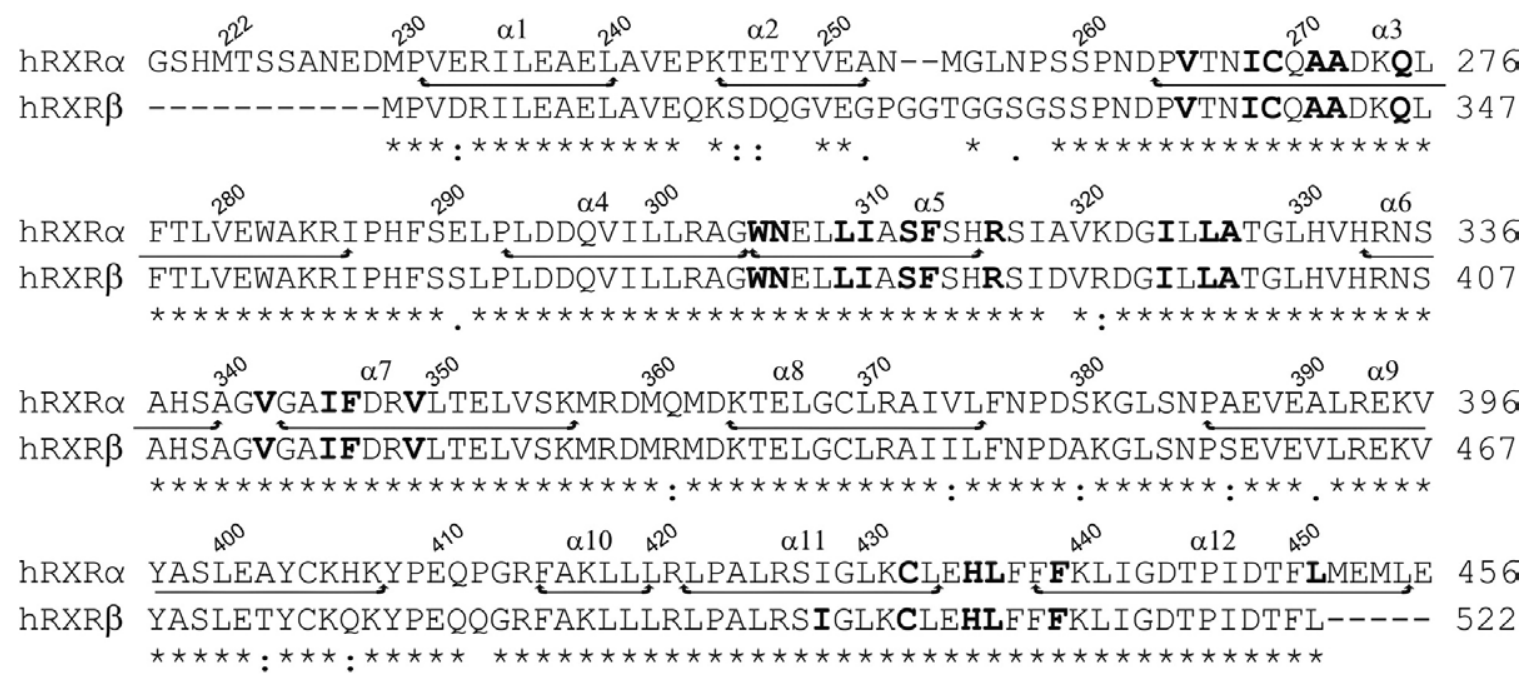

$\mathrm{hRXR} \alpha$ APHQMT 462

$h \operatorname{RXR} \beta------$

Figure 4. Structure resolved complexes LG 100268-hRXR $\beta$ LBD (PDB entry: 1h9u) and 9-cis-RAhRXR $\alpha$ LBD (PDB entry: 1fby) sequence alignment to exhibit the similarities between these two proteins. Bold characters represent the contacted sites for the agonist in each complex based on LPC/CSU program analysis. Residues are numbered according to the human sequence of the protein. The twelve $\alpha$-helical regions are marked for $\mathrm{hRXR} \alpha$ based on their agonist free crystal structure (PDB entry: 1lbd). 
first $2 \mathrm{~min}$ period, but this protection appears to be greater than that observed for 9-cis-RA, again resulting in slower deuterium exchange kinetics. The deuterium incorporation level for this fragment indicated that the methoprenic acid-hRXR $\alpha$ complex, if it existed at all, was only very weakly associated at this site.

Significantly lower deuterium levels were observed in the peptide corresponding to residues $310-319$, as was the case previously observed in the 9-cis-RAhRXR $\alpha$ complex [12]. Three residues, Ser312, Phe313, and Arg316, (Figure 4) in this fragment directly contact the ligand 9-cis-RA in the crystal structure of this complex (PDB entry: 1fby). Two types of protection were observed for deuterium exchange-in by other agonists. First, fenretinide and methoprenic acid showed protection of this fragment similar to that found for 9-cis-RA. Second, deuterium exchange-in to this fragment was even further reduced when LG 100268, SR11246, or DHA was bound to the protein.

Previous SAR studies with synthetic analogs of RXR ligands suggested that cancer prevention was based on drug selectivity for specific retinoid receptor subtypes [17]. Thus, the hydrophobic character of the ligands was increased on the bridge linking the aromatic and cyclohexene rings of these agonist structures. The selectivity for $\mathrm{RXR} \alpha$ was further enhanced through a cyclopropyl ring that was designed into the SR11246 and LG 100268 structures to replace the carbon bridge present in the earlier agonists [17]. The resolved crystal structure of the LG 100268-RXR complex was acquired for the RXR $\beta$ LBD (PDB entry: $1 \mathrm{~h} 9 \mathrm{u}$ ), but since the $\beta$-subtype shares $89 \%$ of its sequence with the protein used in the present study, the binding residues are assumed to be almost the same in both complexes (Figure 4). Therefore, the known interaction between LG 100268, which is specific for RXRs but not RARs (retinoic acid receptors) and $\mathrm{hRXR} \beta \mathrm{LBD}$ can be used as a model for binding of LG 100268 to the hRXR $\alpha$ LBD in the present study. The two carbons in the cyclopropyl ring of the LG 100268 structure mainly contact Leu380 and Ile381 in hRXR $\beta$ LBD (Figure 5a, left), which correspond to Leu309 and Ile310 in hRXR $\alpha$ LBD. In the 9-cis-RA-hRXR $\alpha$ complex the ligand binds through its methyl group and C8, C9 and $\mathrm{C} 10$ to these residues in the receptor (Figure 5a, right). The crystal structure indicates that in addition to these two hRXR $\beta$ residues three others, Ser383, Phe 384, and Arg387, are in contact with the ligand. These correspond to Ser312, Phe313 and Arg316, respectively, in $\mathrm{hRXR} \alpha$ for contacts with ligands LG 100268 and 9-cis-RA, (Figure 5b). The hydrogen bonding status of the amide hydrogens in the peptic fragment corresponding to residues 310-319 in $\mathrm{hRXR} \alpha \mathrm{LBD}$ would be the same as those in its complexes with LG 100268 and 9-cis-RA, as suggested by computational results from the HBPlus program. The reduction in deuterium incorporation in this fragment upon binding agonists follows the order SR11246 and DHA > LG $100268 \gg$ 9-cis-RA, fenretinide, and methoprenic acid (Figure 3). Thus, the trend appears to correlate with the increase in hydro-

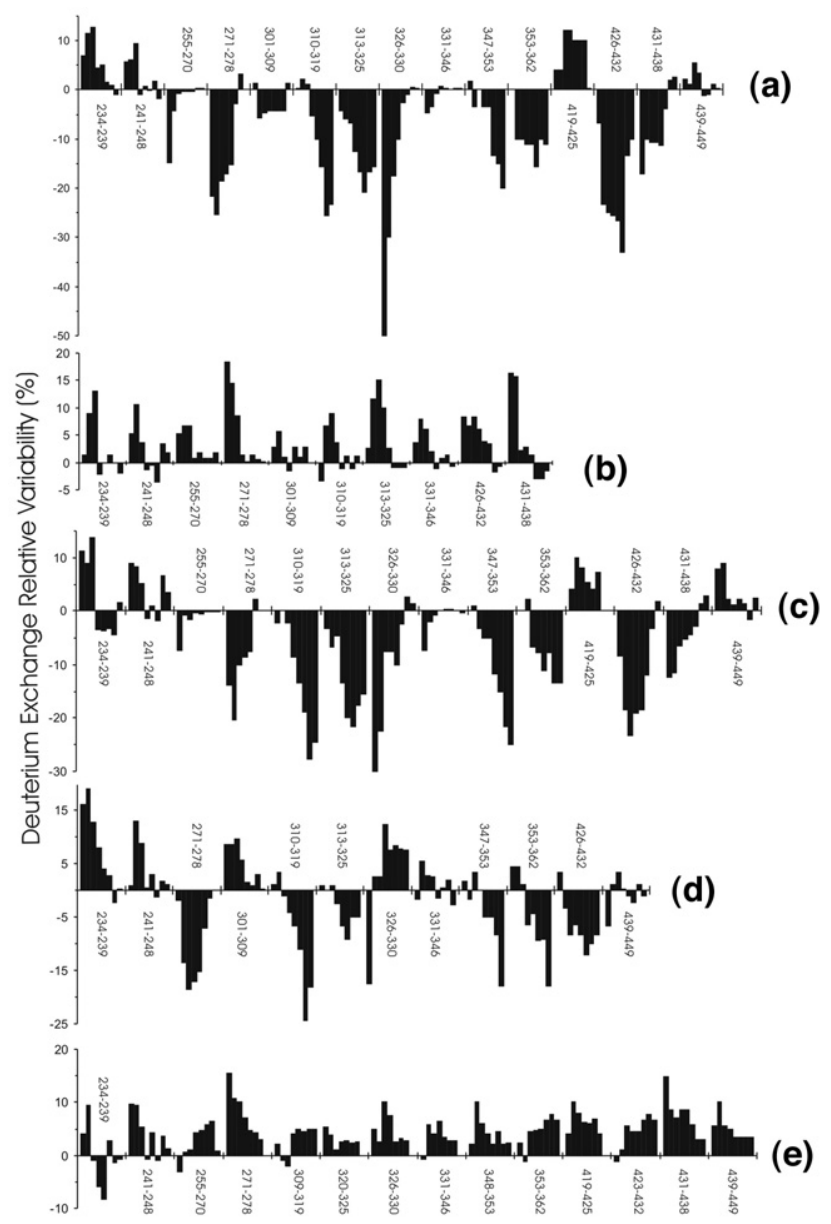

Figure 6. Deuterium exchange-in protection through agonist binding to hRXR $\alpha$ LBD homodimer relative to 9-cis-RA binding as shown in the peptic peptides. The difference in deuterium content between the hRXR $\alpha$-agonist and hRXR $\alpha$-9-cis-RA is exhibited at eight different time points $(0.25,1,2,3,5,10,30$, and $60 \mathrm{~min}$, left to right) for each peptic fragment as a series of vertical bars. The nonprotected regions are not shown (a) LG 100268; (b) fenretinide; (c) SR11246; (d), DHA; (e) methoprenic acid).

phobic interactions inherent in the agonist structures that modified the amide hydrogen bonding network of the interacted regions of the protein and/or shield the protein amide hydrogens from the solvent.

Similarly, reduced deuterium incorporation was observed in the fragment corresponding to residues 431-438, which also contains three X-ray crystallographic contact residues, Cys432, His435, and Leu436 (Figure 4). However, lower protection against H/D exchange appears only in the early time points for 9-cis-RA and most of the agonists, in the complexes with $\mathrm{hRXR} \alpha$. The initial increased protection in this peptide as well as in peptide 271-278 towards deuterium exchange-in by different agonists relative to that observed in peptide 310-319, shows that agonists affect the exchange rates differently in different regions of the protein. The agonist methoprenic acid exhibited limited or no H/D exchange protection in fragment 431-438. LG 100268 exhibited the greatest 
protection at the early time points and fenretinide and DHA showed extended protection over time (Figure 3). In peptic fragment 419-425, the agonists generally showed less protection towards deuterium exchange-in compared with 9-cis-RA, and a generally constant kinetic pattern of exchange over the time course that revealed a lower degree of conformational perturbation for different agonists compared with 9-cis-RA. Overlapping fragments may help to increase the resolution for locating the conformationally changed sites. In the case of methoprenic acid, the fragment corresponding to residues 347-353 from the complex showed no deuterium level changes compared with that from the 9-cis-RA complex, but the deuterium level increased in the fragment corresponding to residues $348-353$. These results indicate that amide hydrogens near Arg348, which is surrounded by three residues that interact with bound agonist (Figure 4), is less protected by methoprenic acid than by 9 -cis-RA.

Analysis of differences in deuterium content in the homodimer $\mathrm{hRXR} \alpha \mathrm{LBD}$ resulting from complexation with 9-cis-RA and that found with other agonists further reveals the comparative rates of amide hydrogen exchange that are affected by different ligands (Figure 6). These exchange differences vary from one region of the protein to another and indicate different types of ligand-induced conformational ensembles. An analysis of the effects of each agonist on amide hydrogen exchange in different sequences of the receptor is facilitated by relating the exchange behavior to the native ligand 9-cis-RA (Figure 6). This comparison immediately reveals the overall effectiveness of the agonists at protecting against deuterium exchange-in. Thus, throughout most of the ligand-binding domain, it is immediately obvious that agonists LG 1000268, SR11246, and DHA are superior to 9-cis-RA at protecting the receptor against amide hydrogen exchange, whereas fenretinide and methoprenic acid are generally seen to be less effective. All of the peptic fragments except those within the sequence $234-248$ contain one or more ligand-binding residues (Figure 4). The results for these fragments also reveal whether a given agonist when compared with 9-cis-RA affects deuterium exchange-in and thus the conformational ensembles in a given region of the protein.

The $\alpha$-helix 12 is a key structural motif for cellular function and regulation in RXR and exhibits extreme flexibility as reveled in both a previous study[12] and the present studies. Despite the dramatic movement of this segment upon binding by 9-cis-RA, the H/D exchange kinetics were essentially unaffected and no deuterium exchange difference was observed in helix 12 in the presence or absence of 9-cis-RA. A change in $\mathrm{H} / \mathrm{D}$ exchange kinetics in this segment, however, was observed on complexation by the other agonists (Figure 6). For example, the N-terminal region of helix 12, which was represented by the fragment corresponding to residues 439-449 apparently showed increased deu- terium incorporation when RXR associated with methoprenic acid. A slight increase in deuterium incorporation was also observed in this fragment upon binding by LG 100268 and SR11246. H/D exchange kinetics in the C-terminal region of helix 12 was not modified by any of these agonists (data not shown), which is consistent with the structural analysis in that agonists do not strongly affect the conformation of the C-terminal region [18]. This unique behavior of helix 12 in deuterium exchange incorporation in the complexes with LG 100246 and SR11246 may be related to the altered positions of helix 12, which resulted in RXR's failure to release the corepressors. No direct information is available for methoprenic acid regarding corepressor release although the observation that the retinoic acid metabolite acts as an agonist in cultured cells [1] suggest that corepressor-coactivator exchange does take place in vivo.

Structurally similar agonists, like LG 100268 and SR11246, which differ only in a methyl group in one aromatic ring and a pyridine ring substituted for a benzene ring (Figure 1), result in very similar amide deuterium exchange kinetic patterns throughout the protein backbone (Figure 6a and c). Clearly, in comparison with the ligand 9-cis-RA, these two agonists cause a significant decrease in the deuterium incorporation in almost all of the $\operatorname{hRXR} \alpha$ LBD H/D exchanging sites. Since all ligands have dissociation constants in the nM range, they are saturating at $\mu \mathrm{M}$ concentrations of protein and ligand. Thus, the protection may arise from an increased complex lifetime, increased hydrogen bonding to exchangeable backbone amides, an altered protein conformation, or a protein conformation unique to these ligands. The deuterium incorporation of fenretinide or methoprenic acid shows some similarity to that of 9-cis-RA as ligand, the most important of which is that in almost all peptides from the $\mathrm{hRXR} \alpha \mathrm{LBD}$, the amide deuterium levels were greater. Structurally, there also are similarities in these two compounds, most notably the isoprenoid motif that serves as the backbone. RXR is reportedly activated in human cells by this metabolite of the pesticide, methoprene [19], and high doses have been shown to cause teratogenic effects during mouse embryogenesis [20]. Despite the results from previous binding studies and the structural analyses performed with the $h R X R \beta$ complex [18], the above results suggest that this ligand poorly protects the $h R X R \alpha$ binding site amide hydrogens from exchange. The reason for this difference is not immediately obvious, but it may be due either to poor affinity under the buffering conditions employed or to interactions occurring at other sites in $\operatorname{hRXR} \alpha$.

\section{Conclusions}

Binding specificities of various agonists in the human $\mathrm{RXR} \alpha$ LBD homodimer were found to be located in the segments containing the binding residues for the native 
ligand, 9-cis-retinoic acid, by hydrogen/deuterium exchange and mass spectrometry. The agonist-specific alternations in H/D exchange may reflect conformational specific effects in either amide $\mathrm{H}$-bonding and/or the accessibility of amide protons to bulk solvent in the agonist bound tertiary structure in the $\mathrm{RXR} \alpha$ homodimer. Therefore, the regions in the protein without direct contact to the agonist exhibit fewer or no changes in $\mathrm{H} / \mathrm{D}$ exchange kinetics for the different agonist$\mathrm{hRXR} \alpha$ complexes.

It is worth noting that deuterium exchange kinetic analysis (Figure 6) provides a rapid and facile single time-point method for analyzing the structural basis of protein-ligand binding effects. The analysis associated with fragment 310-319 and others of $h R X R \alpha$ LBD (Figure 3) provides a dramatic demonstration in the differences caused by bound agonists on amide hydrogen exchange. The application of such a method may be useful in analyzing the structural interaction between agonists and retinoid $\mathrm{X}$ receptor.

\section{Acknowledgments}

The authors acknowledge the nucleic acid and protein core and the mass spectrometry core in the OSU Environmental Health Sciences Center (NIH/NIEHS 00210, ES00040, NIH DK060013). They also acknowledge Drs. Pascal Egea and Dino Moras (IGBMC, Illkirch, France) for the generous gift of the expression plasmid for $\mathrm{hRXR} \alpha$ LBD.

\section{References}

1. Mangelsdorf, D. J.; Evans, R. M. The RXR heterodimers and orphan receptors. Cell 1995, 836, 841-850.

2. Szanto, A.; Narkar, V.; Shen, Q.; Uray, I.; Davies, P.; Nagy, L. Retinoid X receptors: X-ploring their (patho)physiological functions. Cell Death Diff. 2004, 11, S126-S143.

3. Rastinejad, F. Retinoid X receptor and its partners in the nuclear receptor family. Curr. Opin. Struct. Biol. 2001, 11, 33-38.
4. Ahuja, H. S.; Szanto, A.; Nagy, L.; Davies, P. J. The retinoid X receptor and its ligands: Versatile regulators of metabolic function, cell differentiation, and cell death. J. Biol. Regul. Homeost. Agents 2003, 17, 29-45.

5. Hede, K. Rexinoids may be ready for prime time in prevention, but challenges remain. J. Natl. Cancer Inst. 2004, 96, 1807-1808.

6. Mukherjee, R.; Davies, P.; Crombie, D.; Bischoff, E.; Cesario, R.; Jow, L.; Hamann, L.; Boehm, M.; Mondon, C.; Nadzan, A.; Paterniti, J. J.; Heyman, R. Sensitization of diabetic and obese mice to insulin by retinoid $X$ receptor agonists. Nature 1997, 386, 407-410.

7. Villarroya, F.; Iglesias, R.; Giralt, M. Retinoids and retinoid receptors in the control of energy balance: Novel pharmacological strategies in obesity and diabetes. Curr. Med. Chem. 2004, 11, 795-805.

8. Vivat-Hannah, V.; Zusi, F. C. Retinoids as therapeutic agents: Today and tomorrow. Mini. Rev. Med. Chem. 2005, 5, 755-760.

9. Egea, P. F.; Mitschler, A.; Rochel, N.; Ruff, M.; Chambon, P.; Moras, D. Crystal structure of the human $\mathrm{RXR} \alpha$ ligand-binding domain bound to its natural ligand: 9-cis retinoic acid. EMBO J. 2000, 19, 2592-601.

10. Hu, X.; Lazar, M. A. The CoRNR motif controls the recruitment of corepressors by nuclear hormone receptors. Nature 1999, 402, 93-96.

11. Love, J. D.; Gooch, J. T.; Benko, S.; Li, C.; Nagy, L.; Chatterjee, V. K. K.; Evans, R. M.; Schwabe, J. W. R. The structural basis for the specificity of retinoid-X receptor-selective agonists: New insights into the role of helix H12. J. Biol. Chem. 2002, 277, 11385-11391.

12. Yan, X.; Broderick, D.; Leid, M. E.; Schimerlik, M. I.; Deinzer, M. L. Dynamics and ligand-induced solvent accessibility changes in human retinoid X receptor homodimer determined by hydrogen deuterium exchange and mass spectrometry. Biochemistry 2004, 43, 909-917.

13. Sobolev, V.; Sorokine, A.; Prilusky, J.; Abola, E. E.; Edelman, M. Automated analysis of interatomic contacts in proteins. Bioinformatics $1999,15,327-332$.

14. Kabsch, W.; Sander, C. Dictionary of protein secondary structure: Pattern recognition of hydrogen-bonded and geometrical features. Biopolymers 1983, 22, 2577-2637.

15. McDonald, I. K.; Thornton, J. M. Satisfying hydrogen bonding potential in proteins. J. Mol. Biol. 1994, 238, 777-793.

16. Wang, L.; Pan, H.; Smith, D. L. Hydrogen exchange-mass spectrometry. Mol. Cell. Porteom. 2002, 1, 132-138.

17. Dawson, M. I.; Zhang, X. Discovery and design of retinoic acid receptor and retinoid $X$ receptor class- and subtype-selective synthetic analogs of all-trans-retinoic acid and 9-cis-retinoic acid. Curr. Med. Chem. 2002, 9, 623-637.

18. Svensson, S.; Östberg, T.; Jacobsson, M.; Norström, C.; Stefansson, K.; Hallén, D.; Johansson, I. C.; Zachrisson, K.; Ogg, D.; Jendeberg, L. Crystal structure of the heterodimeric complex of LXR $\alpha$ and RXR $\beta$ ligand-binding domains in a fully agonistic conformation. EMBO J. 2003, 22, 4625-4633.

19. Harmon, M. A.; Boehm, M. F.; Heyman, R. A.; Mangelsdorf, D. J. Activation of mammalian retinoid $X$ receptors by the insect growth regulator methoprene. Proc. Natl. Acad. Sci. U.S.A. 1995, 92, 61576160 .

20. Unsworth, B.; Hennen, S.; Krishnakumaran, A.; Ting, P.; Hoffman, N. Teratogenic evaluation of terpenoid derivatives. Life Sci. 1974, 15, $1649-1655$. 